Revue d'histoire de l'Amérique française

REVUE D.HISTOIRE DE L'AMÉRIQUE FRANÇAISE

\title{
Religions et sciences sociales
}

Un chassé-croisé interprétatif entre histoire, théologie et sociologie

\section{Gilles Routhier, Marlene Shore et Jean-Philippe Warren}

Volume 57, numéro 3, hiver 2004

URI : https://id.erudit.org/iderudit/009593ar

DOI : https://doi.org/10.7202/009593ar

Aller au sommaire du numéro

Éditeur(s)

Institut d'histoire de l'Amérique française

ISSN

0035-2357 (imprimé)

1492-1383 (numérique)

Découvrir la revue

Citer cet article

Routhier, G., Shore, M. \& Warren, J.-P. (2004). Religions et sciences sociales : un chassé-croisé interprétatif entre histoire, théologie et sociologie. Revue d'histoire de l'Amérique française, 57(3), 365-369.

https://doi.org/10.7202/009593ar d'utilisation que vous pouvez consulter en ligne. 


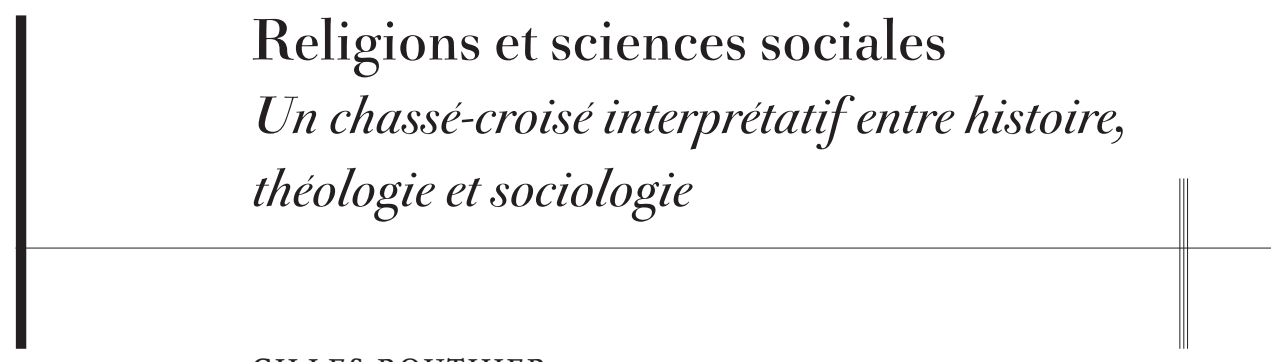

\author{
GILLES ROUTHIER \\ Faculté de théologie \\ Université Laval \\ MARLENE SHORE \\ Département d'histoire \\ York University \\ JEAN-PHILIPPE WARREN \\ Département de sociologie et d'anthropologie \\ Université Concordia
}

ES TROIS ARTICles du présent dossier, rédigés l'un par une historienne,
l'autre par un théologien et le dernier par un sociologue, furent à l'origine des conférences données dans le cadre d'un congrès organisé par l'Institut d'histoire de l'Amérique française sous le thème "Science(s) et culture(s)", et plus spécifiquement dans le cadre d'un atelier intitulé «À la croisée des discours : la religion et la construction du savoir social». En appelant à dialoguer des universitaires formés dans des cultures et des disciplines différentes, ils permettent de mieux comprendre à quel point l'imperméabilité des frontières entre sciences sociales et religions, d'une part, entre Canada anglophone et Canada francophone, d'autre part et, enfin, entre catholicisme et protestantisme, est en partie illusoire. Dit en d'autres termes, ils permettent de mieux comprendre à quel point la "question des frontières » est moins simple qu'on n'a voulu l'imaginer jusqu'ici.

Les relations entre sciences sociales et religions ont trop longtemps été perçues comme contre-nature. Depuis Auguste Comte, lequel faisait de l'âge théologique le premier stade d'une histoire de l'humanité se terminant avec 
l'état positif, il semblait naturel de penser que la modernité des premières et le caractère traditionnel des secondes ne pouvaient faire bon ménage. $\mathrm{Au}$ Québec francophone autant qu'au Canada anglophone, plusieurs historiens des sciences ont repris ce schéma simpliste pour décrire la sortie de la science des limbes de la théologie et de la philosophie morale, opposant, comme l'auraient fait certains philosophes positifs issus d'un autre siècle, la certitude du dogme et le questionnement de la raison.

Ces trois textes n'invalident pas complètement une telle manière de penser le rapport entre religions et sciences sociales. En effet, maintes résistances, exprimées tout au long de la première moitié $\mathrm{du} \mathrm{xx}^{\mathrm{e}}$ siècle par les milieux religieux protestants et catholiques canadiens, ont accompagné l'institutionnalisation des sciences sociales. Les questions sociales étant morales, il revenait aux Églises, déclaraient plusieurs, d’en énoncer les réponses en élaborant une économie politique tout entière fondée sur les Évangiles. Léon Gérin, par exemple, devra sans cesse défendre la légitimité d'une analyse monographique du milieu devant ceux qui s'attachaient à défendre une connaissance a priori, morale et prosélyte de la réalité sociale; et, dans le monde anglophone, une frange du clergé protestant et catholique continuera longtemps à se méfier d'une "sociologie païenne» qui cherchait à remplacer Dieu comme unique auteur de l'ordre social. Reste que la présentation que les trois études publiées ici font de la rencontre entre christianisme et sciences sociales au Canada au cours de la première moitié du siècle dernier lève un voile sur une histoire autrement plus riche et plus complexe.

La mise en perspective nous enseigne d'abord que les milieux libéraux ont été tout autant - sinon davantage — hostiles à l'établissement de la science nouvelle. L'éthique individualiste et volontariste sur laquelle reposait la vision du monde libérale s'opposait de front à une éthique sociologique fondée sur le "solidarisme» et l'acceptation d'une responsabilité collective telle qu'elle s'exprimait à travers les travaux et les engagements des intellectuels chrétiens sensibles aux thèmes ouvriéristes. On retiendra, parmi ces figures pionnières, les noms de James Shaver Woodsworth, dont les ouvrages Strangers within Our Gates (1909) et My Neighbour (1911), peuvent être inclus dans une histoire de la discipline sociologique, de Herbert Brown Ames, auteur de City Below the Hill (1897), ainsi que de Stanilas Lortie, qui fit paraître son Compositeur typographe de Québec dans la troisième série des "Ouvriers des deux mondes» (1904).

Méfiants envers la pensée marxiste, la vaste majorité de ces intellectuels se rattachaient aux courants du social gospel et du catholicisme social qui 
emportaient l'adhésion de plus en plus de chrétiens au tournant du $\mathrm{xx}^{\mathrm{e}}$ siècle. Dans un livre phare sur cette période, paru il y a déjà plus de trente ans, Richard Allen a démontré comment la "passion sociale» de cette période surgissait d'une nouvelle philosophie religieuse qui légitimait désormais l'engagement des chrétiens dans le monde et les incitait à croire en l'établissement possible du royaume de Dieu sur terre ${ }^{1}$. Plusieurs autres études fraîchement publiées tendent à confirmer le rôle de premier plan qu'ont joué les Églises dans la formation, autour des années 1890-1910, d'un nouveau paradigme idéologique, paradigme ayant permis l'institutionnalisation des sciences sociales, et de la sociologie en particulier, dans les établissements d'enseignement supérieur, dans les différentes institutions religieuses et dans la fonction publique.

Dans les limites de leur théologie, selon les traditions de pensée qui leur étaient propres (les Églises anglicane, méthodiste, baptiste, catholique, etc., représentant autant d'interprétations particulières d'une même volonté de réforme et de direction de la société), les Églises protestantes et catholique n'ont pas entièrement boudé l'étude et la pratique des sciences sociales, ce que tendent à démontrer plusieurs études parues récemment. Que ce soit dans le déjà ancien The Regenerators : Social Criticism in Late Victorian English Canada, ou le bien documenté A Full-Orbed Christianity: The Protestant Churches and Social Welfare in Canada, 1900-1940, ou le passionnant Quand la jeunesse entre en scène: l'Action catholique avant la Révolution tranquille, ou encore le magistral Matters of Mind: The University in Ontario, 1791-1951, pour ne citer ici que ces quatre volumes parmi une littérature devenue soudain (c'est-à-dire depuis dix ans) abondante, on retrouve le portrait d'Églises plus dynamiques et plus engagées socialement par rapport à l'image dépeinte par les auteurs des années 1970 et $1980^{2}$.

Pris ensemble, ces trois articles mettent en lumière, ce qu'on n'a pas assez fait jusqu'ici, les nombreux parallèles entre le cheminement des sciences sociales au Canada anglophone et au Canada francophone. L'article de Marlene Shore, qui porte sur l'institutionnalisation de la sociologie à

1. Richard Allen, The Social Passion : Religion and Social Reform in Canada, 1914-1928 (Toronto, University of Toronto Press, 1971).

2. Ramsay Cook, The Regenerators : Social Criticism in Late Victorian English Canada (Toronto, University of Toronto Press, 1985); Nancy Christie et Michael Gauvreau, A Full-Orbed Christianity: The Protestant Churches and Social Welfare in Canada, 1900-1940 (Montréal, McGill-Queen University Press, 1996); Louise Bienvenue, Quand la jeunesse entre en scène: l'Action catholique avant la Révolution tranquille (Montréal, Boréal, 2003). Brian McKillop, Matters of Mind: The University in Ontario, 1791-1951 (Toronto, University of Toronto Press, 1994). 
l'Université McGill, permet justement, par le jeu des comparaisons, de mieux apprécier cette réalité historique trop souvent occultée dans certains travaux «nationalistes». On dira que la chose s'entend aisément, puisque, d'un bord et de l'autre de l'Outaouais, les praticiens des sciences de l'Homme étaient aux prises avec la tâche de répondre à des défis semblables, ceux liés au rapide progrès économique et social d'une nation en plein essor. Mais il faudrait pousser cette première réponse plus loin, et envisager aussi ces parallèles comme le résultat de traditions intellectuelles dont les foyers épistémiques, quoique pluriels, ne sont pas moins en partie convergents. C'est en ce sens que l'on peut dire qu'une étude plus approfondie de l'histoire des sciences sociales au Canada pourrait aider au dialogue des "deux solitudes» en analysant de manière plus fine comment le Canada anglophone et le Canada francophone ont fait face aux problèmes de l'industrialisation, de l'urbanisation et de la démocratisation dans la première moitié du $\mathrm{xx}^{\mathrm{e}}$ siècle.

Alors que, avec le siècle, les sciences sociales sont de plus en plus instituées dans les programmes universitaires et progressivement dissociées de leurs premières filiations religieuses, elles ne cessent pas de porter en elles les préoccupations morales et sociales du monde religieux, quoique désormais sur une base toute différente. Il n'est certes pas étonnant par conséquent que, durant l'entre-deux-guerres, l'élite des affaires et l'élite universitaire aient pu craindre la critique sociale proposée par les sciences sociales, puisque une telle critique s'articulait sur une condamnation du «monde» et sur un prophétisme dont le radicalisme prenait racine dans un tuf religieux, catholique ou protestant.

Ce qu'il reste maintenant à comprendre, c'est comment la sécularisation croissante de la société canadienne a pu être encouragée par les mouvements religieux, et comment ceux-ci ont pu contribuer ainsi à la marginalisation des Églises comme force politique et sociale. À Harold Innis qui écrivait : «The skyscraper has become the modern cathedral; long-term credit is the new basis of modern belief», McKillop répliquait : «Secularization indeed took place, but its locus was less in any arithmetic decline in church membership or increase in "unbelief" than it was in the directions taken by those whose spiritual need to "connect" with others took them outside church walls and into the occupational structures of "secular" society itself"." On ne saurait si bien dire. Mais encore faut-il comprendre les tenants et

3. A. Brian McKillop, A Disciplined Intelligence. Critical Inquiry and Canadian Thought in the Victorian Era (Montréal, McGill-Queen’s University Press, 2001), xxvi. 
aboutissants de cette «externalisation» qui a élargi l'aire d'action des Églises dans le même temps où elle signait la fin de leur puissance et de leur influence. Selon une vue sommaire, on pourrait avancer que la recherche des moyens grâce auxquels serait assurée la rédemption de l'Homme et de la société a conduit, à partir de l'après-guerre, à l'évincement des fins que les Églises chrétiennes proposaient aux consciences individuelles et à la collectivité humaine. Peu importe, toutefois, l'hypothèse adoptée. L’important n'est-il pas que le débat soit lancé?

Le dialogue entre ces trois articles nous conduit à quelques conclusions provisoires. Il permet de mieux saisir la complexité de la relation entre christianisme et sciences sociales, une relation qui ne peut plus être pensée en termes d'exclusion réciproque. Ces articles font aussi la démonstration de la fécondité des approches comparatives. De manière prospective - et à l'intention des jeunes chercheurs - ils manifestent la nécessité de reprendre à nouveau certains dossiers historiques afin de comprendre la sécularisation, non comme un mouvement issu de l'extérieur du christianisme, mais comme un mouvement plongeant ses racines au cœur même de celui-ci. 